\title{
A Method to Evaluate Quantum Path Integrals
}

\author{
Babak Vakili \\ Department of Basic Sciences, Tonekabon Branch, Islamic Azad University (IAU), Tonekabon, Iran \\ Email: b-vakili@iauc.ac.ir
}

\begin{abstract}
As an alternative formulation of quantum mechanics, path integral is based on the notion of transition amplitude which gives the wave function of a quantum system at a time $t_{f}$ by acting on the wave function at an earlier time $t_{i}$. We show that for a general quadratic form for the Lagrangian of the system, transition amplitude has the form $f\left(t_{f}-t_{i}\right) e^{\frac{i}{\hbar} S_{c l a s s}}$, where $S_{\text {class. }}$ is the classical action. We then present an algebraic method to evaluate the function $f\left(t_{f}-t_{i}\right)$ without refereing to the path integral calculations. We examine the presented method to the cases of free particle and harmonic oscillator and obtain their propagators.
\end{abstract}

Keywords: Path integral, classical action.

\section{Introduction}

The formulation of quantum mechanics is based on the commutation relations between the conjugate variables $(q, p)$, as $[q, p]=i \hbar$. In Heisenberg picture the coordinate (or any other) operator at time $t$ is given by $q(t)=e^{i / \hbar H t} q_{0} e^{-i / \hbar H t}$, where $H$ is the Hamiltonian operator and $q_{0}$ is the coordinate operator at time $t=0$. Now, if one denotes the eigenstates of the operator $q_{0}$ with $\mid q^{\prime}>$ corresponds to the eigenvalue $q^{\prime}$, then eigenstates of $q\left(t^{\prime}\right)$ with eigenvalue $q^{\prime}$ will be $\left|q^{\prime}, t^{\prime}>=e^{\frac{i}{\hbar} H t}\right| q^{\prime}>$. According to the basic principles of quantum mechanics a quantum system can be completely described by the transition amplitude (propagator) $<q^{\prime \prime}, t^{\prime \prime}\left|q^{\prime}, t^{\prime}>=<q^{\prime \prime}\right| e^{-\frac{i}{\hbar} H\left(t^{\prime \prime}-t^{\prime}\right)} \mid q^{\prime}>$. One of the most powerful methods to evaluate this amplitude is the Feynman path integral through which the above propagator can be expressed in terms of the classical Lagrangian of the system without any references to the operators and Hilbert space [1]. The main idea in relation between the quantum propagators and the classical characters of a system returned to the Dirac's work [2], according to which the amplitude should be proportional to $e^{\frac{i}{\hbar} S_{c l a s s}}$. This statement had been justified later by the Feynman's path integral formulation of quantum mechanics.

In this letter after a brief review on the subject, we introduce the amplitude formula for the free particle and harmonic oscillator and show that they have the form $f(T) e^{\frac{i}{\hbar} S\left[q_{c l}\right]}$, where $T$ is the time interval between the initial and final states. Then, in section 3, we will introduce a wide class of Lagrangians for which the propagator can be written of this form. At the end of this section we present a path integral formula to evaluate the function $f(T)$. In section 4 , a systematic algebraic method is introduced to obtain the function $f(T)$ without reference to the path integral calculations. Section 5 is devoted to the summary and conclusions.

\section{Path Integral and Propagators: a Brief Review}

It is well-known that the wave function in Schrödinger equation at an arbitrary time $t$ can be obtained from the action of the time evolution operator $G\left(t, t_{0}\right)$ on the wave function at some initial time $t_{0}$

$$
\left|\Psi(t)>=G\left(t, t_{0}\right)\right| \Psi\left(t_{0}\right)>.
$$

For a time-independent Hamiltonian we have

$$
G\left(t, t_{0}\right)=\theta\left(t-t_{0}\right) e^{-\frac{i}{\hbar}\left(t-t_{0}\right) H},
$$


where $\theta\left(t-t_{0}\right)$ is the Heaviside step function. It is clear that this operator is nothing but the Green function for the time-dependent Schrödinger equation, that is

$$
\left(i \hbar \frac{\partial}{\partial t}-H\right) G\left(t, t_{0}\right)=i \hbar \delta\left(t-t_{0}\right) \text {. }
$$

Having the function $G$, one can evaluate its matrix elements in a given basis. Therefore, in coordinate basis $X|x\rangle=x \mid x>$, we have $\left\langle x\left|G\left(t, t_{0}\right)\right| x_{0}>=G\left(t, x ; t_{0}, x_{0}\right)\right.$. If we have the function $G\left(t, x ; t_{0}, x_{0}\right)$, then the time evolution of the Schrödinger wave function can be written as

$$
\Psi(x, t)=\int \mathrm{d} x^{\prime} G\left(t, x ; t^{\prime}, x^{\prime}\right) \Psi\left(x^{\prime}, t^{\prime}\right) .
$$

It should be noted that the dependence on the intermediate times drops out in the above equation. In Schrödinger picture the quantum states $\mid \Psi(t)>$ carry time dependence while the operators are time independent. In Heisenberg picture, on the other hand, quantum states are time-independent such that we can identify a Heisenberg state $\mid \Psi>_{H}$ with its Schrödinger counterpart $\mid \Psi(t)>_{S}$ with $\mid \Psi>_{H}=$ $e^{\frac{i}{\hbar} t H} \mid \Psi(t)>_{S}$. In this picture the operators carry all time dependence in such a way that an operator in Heisenberg picture is related to its Schrödinger picture through the relation $A_{H}(t)=e^{\frac{i}{\hbar} t H} A e^{-\frac{i}{\hbar} t H}$. For example, the eigenstates of the position operator satisfying $X_{H}(t)\left|x, t>_{H}=x\right| x, t>_{H}$, are then easily seen to be related to the coordinate basis in Schrödinger picture as $\left|x, t>_{H}=e^{\frac{i}{\hbar} t H}\right| x>$. Now, it is easy to see that for $t>t_{0}$, we have

$$
\begin{array}{r}
H<x, t\left|x_{0}, t_{0}>_{H}=<x\right| e^{-\frac{i}{\hbar} t H} e^{\frac{i}{\hbar} t_{0} H}\left|x_{0}>=<x\right| e^{-\frac{i}{\hbar}\left(t-t_{0}\right) H} \mid x_{0}> \\
=<x\left|G\left(t, t_{0}\right)\right| x_{0}>=G\left(t, x ; t_{0}, x_{0}\right) .
\end{array}
$$

Therefore, we see that the matrix elements of the time evolution operator is indeed the time ordered transition amplitude between the coordinates basis states in the Heisenberg pictures. In path integral picture to evaluate the transition amplitude, one usually divides the time interval between the initial and final time into $N$ equal segments of infinitesimal length $\epsilon$ as $\epsilon=\left(t_{f}-t_{i}\right) / N$ and in the end takes the continuum limit $\epsilon \rightarrow 0$ and $N \rightarrow \infty$. In this set-up we can write

$$
\begin{gathered}
G\left(t_{f}, x_{f} ; t_{i}, x_{i}\right)=_{H}<x_{f}, t_{f} \mid x_{i}, t_{i}>_{H}= \\
\lim _{\epsilon \rightarrow 0, N \rightarrow \infty} \int \mathrm{d} x_{1} \ldots \mathrm{d} x_{N-1}<x_{f}, t_{f}\left|x_{N-1}, t_{N-1}>\ldots<x_{1}, t_{1}\right| x_{i}, t_{i}>,
\end{gathered}
$$

in which we have used $\int \mathrm{d} x|x, t><x, t|=1$. Also, all of the intermediates kets are in Heisenberg picture and we have assumed an inherent time ordering from left to right. A straightforward calculation can show that for the systems with Hamiltonian $H=p^{2} / 2 m+V(x)$ the above formula takes the form [3]

$$
\begin{gathered}
G\left(t_{f}, x_{f} ; t_{i}, x_{i}\right)= \\
\lim _{\epsilon \rightarrow 0, N \rightarrow \infty}\left(\frac{m}{2 \pi i \hbar \epsilon}\right)^{N / 2} \int \mathrm{d} x_{1} \ldots \mathrm{d} x_{N-1} \exp \left\{\frac{i \epsilon}{\hbar} \sum_{n=1}^{N}\left[\frac{m}{2}\left(\frac{x_{n}-x_{n-1}}{\epsilon}\right)^{2}-V\left(\frac{x_{n}+x_{n+1}}{2}\right)\right]\right\} .
\end{gathered}
$$

A glance at the expression under the exponential function shows that in the continuum limit it tends to the expression $\frac{i}{\hbar} \int_{t_{i}}^{t_{f}} d t\left(\frac{1}{2} m \dot{x}^{2}-V(x)\right)$, which in turn is nothing other than the action $S[x]$ of the system. Thus, we are led to the following well-known expression for the path integral

$$
G\left(t_{f}, x_{f} ; t_{i}, x_{i}\right)=A \int \mathcal{D} x e^{\frac{i}{\hbar} S[x]},
$$

where $A$ is a constant independent of the dynamics of the system.

It is seen that the path integral is a functional integral, namely, the integrand which is the phase factor is a functional of the trajectory between the initial and the final points. Although, in general the evaluation of such an integral may be a difficult task, its evaluation for the simple systems can be found in any text books of quantum mechanics. For example, in the case of a one dimensional free particle whose action is $S[x]=\int_{t_{i}}^{t_{f}} d t \frac{1}{2} m \dot{x}^{2}$, the result is

$$
G\left(t_{f}, x_{f} ; t_{i}, x_{i}\right)=\left[\frac{m}{2 \pi i \hbar\left(t_{f}-t_{i}\right)}\right]^{1 / 2} \exp \left[\frac{i}{\hbar} \frac{1}{2} m \frac{\left(x_{f}-x_{i}\right)^{2}}{\left(t_{f}-t_{i}\right)}\right] .
$$


Noting that for a free particle we have $\dot{x}=v=\frac{x_{f}-x_{i}}{t_{f}-t_{i}}$, one immediately concludes that in terms of the classical trajectory for which we have $\dot{x_{c l}}=v$, the action takes the form

$$
S\left[x_{c l}\right]=\frac{1}{2} m \frac{\left(x_{f}-x_{i}\right)^{2}}{\left(t_{f}-t_{i}\right)} .
$$

Therefore, the expression (9) will be simplified as

$$
G\left(t_{f}, x_{f} ; t_{i}, x_{i}\right)=\left[\frac{m}{2 \pi i \hbar T}\right]^{1 / 2} e^{\frac{i}{\hbar} S\left[x_{c l}\right]}
$$

where $T=t_{f}-t_{i}$. Another system for which the path integral (8) can be analytically evaluated is the one dimensional harmonic oscillator with action $S[x]=\int_{t_{i}}^{t_{f}} d t \frac{1}{2} m\left(\dot{x}^{2}-\omega^{2} x^{2}\right)$. In this case calculations yield to

$$
G\left(t_{f}, x_{f} ; t_{i}, x_{i}\right)=\left[\frac{m \omega}{2 \pi i \hbar \sin \omega T}\right]^{1 / 2} e^{\frac{i}{\hbar} S\left[x_{c l}\right]}
$$

which reduces to the transition amplitude for the free particle in the limit $\omega \rightarrow 0$. We see from above two examples that in both cases the transition amplitude has the form

$$
G\left(t_{f}, x_{f} ; t_{i}, x_{i}\right)=f(T) e^{\frac{i}{\hbar} S\left[x_{c l}\right]},
$$

where $f(T)$ is a function of $T=t_{f}-t_{i}$. In the next section we are going to present a wide class of dynamical systems whose transition amplitudes have the form of the relation (13) and then will give a systematic method to find the function $f(T)$.

\section{The Model}

In this section we consider a dynamical system with action

$$
S[q]=\int_{t_{i}}^{t_{f}} L(q, \dot{q}) \mathrm{d} t
$$

in which the Lagrangian function has the form [4]

$$
L(q, \dot{q})=a(t) \dot{q}^{2}+b(t) \dot{q} q+c(t) q^{2}+d(t) \dot{q}+e(t) q .
$$

Variation the above action with respect to $q$ shows that the classical trajectory satisfies the following equation

$$
2 a \ddot{q_{c l}}+2 \dot{a} \dot{q}_{c l}+(\dot{b}-2 c) q_{c l}+\dot{d}-e=0 .
$$

To evaluate the propagator $G\left(t_{f}, q_{f} ; t_{i}, q_{i}\right)=A \int \mathcal{D} q e^{\frac{i}{\hbar} S[q]}$, let us use the change of variable

$$
q(t)=q_{c l}(t)+\eta(t)
$$

in which $\eta(t)$ is a variation around the classical path $q_{c l}$ and vanishes at the end points: $\eta\left(t_{i}\right)=\eta\left(t_{f}\right)=0$. Under this change of path, the propagator takes the form

$$
G\left(t_{f}, q_{f} ; t_{i}, q_{i}\right)=A \int \mathcal{D} \eta e^{\frac{i}{\hbar} S\left[q_{c l}+\eta\right]},
$$

where

$$
S\left[q_{c l}+\eta\right]=\int_{t_{i}}^{t_{f}} \mathrm{~d} t\left[a\left(\dot{q_{c l}}+\dot{\eta}\right)^{2}+b\left(\dot{q_{c l}}+\dot{\eta}\right)\left(q_{c l}+\eta\right)+c\left(q_{c l}+\eta\right)^{2}+d\left(\dot{q_{c l}}+\dot{\eta}\right)+e\left(q_{c l}+\eta\right)\right] .
$$


It is easy to show that the above integral can be rewritten in the form

$$
\begin{array}{r}
S\left[q_{c l}+\eta\right]=S\left[q_{c l}\right]+\int_{t_{i}}^{t_{f}} \mathrm{~d} t\left(a \dot{\eta}^{2}+b \dot{\eta} \eta+c \eta^{2}\right) \\
+\int_{t_{i}}^{t_{f}} \mathrm{~d} t\left[\dot{\eta}\left(2 a \dot{q_{c l}}+b q_{c l}+d\right)+\eta\left(b \dot{q_{c l}}+2 c q_{c l}+e\right)\right],
\end{array}
$$

where

$$
S\left[q_{c l}\right]=\int_{t_{i}}^{t_{f}} \mathrm{~d} t\left(a{\dot{q_{c l}}}^{2}+b q_{c l} \dot{q_{c l}}+c q_{c l}^{2}+d \dot{q_{c l}}+e q_{c l}\right) .
$$

Now, let us focus on the last term of (20). By integration by parts and noting that $\eta\left(t_{i}\right)=\eta\left(t_{f}\right)=0$, this term takes the form

$$
-\int_{t_{i}}^{t_{f}} \mathrm{~d} t\left[2 a \ddot{q_{c l}}+2 \dot{a} \dot{q_{c l}}+(\dot{b}-2 c) q_{c l}+\dot{d}-e\right] \eta(t),
$$

which vanishes due to the equation (16). Therefore, with the help of (20), the expression for the propagator (18) takes the form

$$
G\left(t_{f}, q_{f} ; t_{i}, q_{i}\right)=f\left(t_{i}, t_{f}\right) e^{\frac{i}{\hbar} S\left[q_{c l}\right]},
$$

in which

$$
f\left(t_{i}, t_{f}\right)=A \int \mathcal{D} \eta(t) \exp \left[\frac{i}{\hbar} \int_{t_{i}}^{t_{f}} \mathrm{~d} t\left(a \dot{\eta}^{2}+b \dot{\eta} \eta+c \eta^{2}\right)\right] .
$$

Thus, for any dynamical system whose Lagrangian can be written in the form of the relation (15), the quantum propagator is in the form of (23). In the next section we are going to evaluate the function $f$ by an algebraic method without a direct use of the path integral formula (24).

\section{A Method for Evaluation $f\left(t_{i}, t_{f}\right)$}

As we have seen in the previous section, for a wide class of Lagrangian systems the propagator has the form of relation (23). Therefore, the function $f\left(t_{i}, t_{f}\right)$ plays an important role since all information of the path integral propagator is now encoded in it. Our goal in this section is to present a systematic method to evaluate this function without dealing with the path integral formula (24). As usual let us consider the free particle case. According to a well-known property of the propagators we have

$$
G\left(q_{f}, t_{f} ; q_{i}, t_{i}\right)=\int \mathrm{d} q_{c} G\left(q_{f}, t_{f} ; q_{c}, t_{c}\right) G\left(q_{c}, t_{c} ; q_{i}, t_{i}\right)
$$

For a free particle this relation takes the form

$$
f\left(t_{f}-t_{i}\right) e^{\frac{i m}{2 \hbar} \frac{\left(q_{f}-q_{i}\right)^{2}}{t_{f}-t_{i}}}=\int \mathrm{d} q_{c} f\left(t_{f}-t_{c}\right) f\left(t_{c}-t_{i}\right) \exp \left[\frac{i m}{2 \hbar}\left(\frac{\left(q_{f}-q_{c}\right)^{2}}{t_{f}-t_{c}}+\frac{\left(q_{c}-q_{i}\right)^{2}}{t_{c}-t_{i}}\right)\right],
$$

in which we have used the relation (10). If we examine this relation in the case where $q_{f}=q_{i}=0$, we get

$$
f(t+s)=\int \mathrm{d} q_{c} f(t) f(s) \exp \left[\frac{i m}{\hbar}\left(\frac{1}{t}+\frac{1}{s}\right) q_{c}^{2}\right]
$$

where $t=t_{f}-t_{c}$ and $s=t_{c}-t_{i}$. The above integral can be evaluated with the help of the integration formula $\int e^{i \lambda x^{2}} \mathrm{~d} x=\sqrt{i \pi / \lambda}$, leading to

$$
\tilde{f}(t+s)=\sqrt{\frac{i \pi \hbar}{m}} \widetilde{f}(t) \tilde{f}(s)
$$

in which we have defined $\widetilde{f}(t)=\sqrt{t} f(t)$. It is clear that the functions of the form $\tilde{f}(t)=\sqrt{\frac{m}{i \pi \hbar}} e^{\alpha t}$ satisfy this equation. Therefore, we find the following form for the function $f(t)$

$$
f(t)=\sqrt{\frac{m}{2 \pi i \hbar t}} e^{\alpha t}
$$


In what follows we will see how this method may lead us to the right form for the function $f\left(t_{i}, t_{f}\right)$ which we need in front of the factor $e^{\frac{i}{\hbar} S\left[q_{c l}\right]}$ in the path integral propagator. Inspired of the free particle, our method is based on the three steps:

I) The condition (25) should be satisfied by a propagator.

II) We will show that this condition leads us to an equation of the form

$$
f(t+s)=\mathcal{F}(f(t), f(s)),
$$

which can be transformed to the differential equation

$$
\widetilde{\mathcal{F}}\left(f(t), f^{\prime}(t)\right)=0 .
$$

III) we demand

$$
G\left(q_{f}, t_{f} ; q_{i}, t_{f}\right)=\delta\left(q_{f}-q_{i}\right),
$$

which means that the eigenstates of the position operator are non-degenerate and its eigenvalues are continuous.

At the beginning of this section we saw how applying the step I on a free particle led us to the equation (28) which is of the form of the equations (30) introduced in step II. To see how step III can help us to find the complete form of the solution of (30), let us take a look at the equations of the form $f(t+s)=f(t) f(s)$. Taking $s=\mathrm{d} t$ we have

$$
f(t+\mathrm{d} t)=f(t) f(\mathrm{~d} t)
$$

which upon expanding both sides will transform to

$$
f(t)+\mathrm{d} f(t)=f(t)\left(f(0)+\mathrm{d} t f^{\prime}(0)\right),
$$

from which we get

$$
f(0)=1, \quad \mathrm{~d} f(t)=f^{\prime}(0) f(t) \mathrm{d} t .
$$

This is nothing other than a first order differential equation whose initial condition is coded in itself. With $\alpha=f^{\prime}(0)$ we obtain a class of one parameter solutions as

$$
f(t)=e^{\alpha t}
$$

For the free particle, up to a numerical factor we had

$$
f(t+s)=\sqrt{\frac{t s}{t+s}} f(t) f(s),
$$

which with $s=\mathrm{d} t$ takes the form

$$
f(t+\mathrm{d} t)=f(t) f(\mathrm{~d} t) \sqrt{\left(1-\frac{\mathrm{d} t}{t}\right) \mathrm{d} t} .
$$

Now, let us follow what we have done above and expand both sides of this equation. The result is

$$
f(t)+\mathrm{d} f(t)=f(t)\left(f(0)+f^{\prime}(0) \mathrm{d} t\right)\left(1-\frac{\mathrm{d} t}{2 t}\right) \sqrt{\mathrm{d} t} .
$$

After evaluating the square of the both sides, this equation takes the form

$$
f(t)^{2}+2 f(t) \mathrm{d} f(t)=f(t)^{2} f(0)^{2} \mathrm{~d} t .
$$

A glance at this result shows that while in the left hand side we have an infinitesimal term plus an additional finite quantity, in the right hand side there is only an infinitesimal term. Therefore, we should have $f(0)=\infty$, i.e., the function $f(t)$ should be singular at $t=0$. To see how $f(t)$ behaves near its singular point we consider the following limit 


$$
\lim _{s \rightarrow 0} f(t+s)=\lim _{s \rightarrow 0} \sqrt{\frac{t s}{t+s}} f(t) f(s)
$$

from which we get

$$
f(t)=f(t) \lim _{s \rightarrow 0} f(s) \sqrt{\frac{t s}{t+s}} \Rightarrow \lim _{s \rightarrow 0} f(s)=\lim _{s \rightarrow 0} \sqrt{\frac{t+s}{t s}}=\lim _{s \rightarrow 0} \sqrt{\frac{1}{t}+\frac{1}{s}},
$$

which means that

$$
\lim _{s \rightarrow 0} f(s)=\lim _{s \rightarrow 0} \sqrt{\frac{1}{s}} .
$$

Therefore, when $t \rightarrow 0$ the function $f(t)$ has a behavior as $f(t) \sim \frac{1}{\sqrt{t}}$ such that we may write

$$
f(t)=\frac{1}{\sqrt{t}} \widetilde{f}(t)
$$

Now, from (37) we have $\tilde{f}(t) \tilde{f}(s)=\widetilde{f}(t+s)$ which we have already solved it with solution $\widetilde{f}(t)=e^{\alpha t}$. Thus,

$$
f(t)=\frac{1}{\sqrt{t}} e^{\alpha t}
$$

which up to a numerical factor is the same result as we perviously obtained in (29). Finally, to find the parameter $\alpha$ we should take the third step. For a free particle, by fixing the numerical coefficient this step yields

$$
\delta\left(t_{f}-t_{i}\right)=\lim _{t \rightarrow 0} \frac{1}{\pi t} e^{-\frac{\left(q_{f}-q_{i}\right)^{2}}{t}}=\lim _{t \rightarrow 0} \sqrt{\frac{m}{2 \pi i \hbar t}} e^{\alpha t} e^{-\frac{i m}{2 \hbar} \frac{\left(q_{f}-q_{i}\right)^{2}}{t}} .
$$

Comparing these two limits shows that $\alpha=0$, which finally leads us to

$$
f(T)=\sqrt{\frac{m}{2 \pi i \hbar T}},
$$

for the case of a free particle.

Now, let us see how this method may work for the harmonic oscillator. In this case by the step I we have

$$
f\left(t_{f}-t_{i}\right)=\left\{\int \mathrm{d} q_{c} \exp \left[\frac{i m \omega q_{c}^{2} \cos \omega\left(t_{f}-t_{c}\right)}{2 \hbar \sin \omega\left(t_{f}-t_{c}\right)}\right] \exp \left[\frac{i m \omega q_{c}^{2} \cos \omega\left(t_{c}-t_{i}\right)}{2 \hbar \sin \omega\left(t_{c}-t_{i}\right)}\right]\right\} f\left(t_{f}-t_{c}\right) f\left(t_{c}-t_{i}\right),
$$

in which we have set $q_{f}=q_{i}=0$. With the same notation as before, i.e. $t=t_{f}-t_{c}$ and $s=t_{c}-t_{i}$, this equation becomes

$$
f(t+s)=f(t) f(s) \int \mathrm{d} q_{c} \exp \left[\frac{i m \omega}{2 \hbar} q_{c}^{2}(\cot \omega t+\cot \omega s)\right],
$$

which is a Gaussian integral and can be evaluated as

$$
f(t+s)=f(t) f(s) \sqrt{\frac{2 \pi i \hbar}{m \omega(\cot \omega t+\cot \omega s)}} .
$$

To begin the step II we examine the behavior of the function $f(t)$ when $t \rightarrow 0$. To do this, setting $s=0$ in the above equation gives $f(0)=\infty$ and so like the case of the free particle the function $f(t)$ exhibits a singularity in this limit. Following the limiting process (41)-(43) shows that the behavior of this function in the vicinity of its singular point is

$$
\lim _{s \rightarrow 0} f(s)=\lim _{s \rightarrow 0} \sqrt{\frac{m \omega}{2 \pi i \hbar} \cot \omega s .}
$$


Thus we may write

$$
f(t)=\sqrt{\frac{m \omega}{2 \pi i \hbar} \cot \omega t} \widetilde{f}(t),
$$

which upon institution in the relation (50), after some algebra gives

$$
\frac{\tilde{f}(t+s)}{\widetilde{f}(t) \widetilde{f}(s)}=\frac{1}{\sqrt{1-\tan \omega t \tan \omega s}} .
$$

Now, let us take $s=\mathrm{d} t$ to reach

$$
\frac{\widetilde{f}(t+\mathrm{d} t)}{\widetilde{f}(t) \widetilde{f}(\mathrm{~d} t)}=1+\frac{1}{2} \omega \mathrm{d} t \tan \omega t
$$

which in turn yields to the differential relation

$$
\widetilde{f}(t)+d \tilde{f}(t)=\tilde{f}(t) \tilde{f}(0)+\mathrm{d} t \tilde{f}(t)\left(\tilde{f}(0)+\frac{\omega}{2} \tan \omega t\right) .
$$

Therefore, we are arrived at the following differential equation with initial condition $(\alpha=f(0))$

$$
\widetilde{f}(0)=1, \quad d \ln \tilde{f}(t)=\mathrm{d} t\left(\alpha+\frac{\omega}{2} \tan \omega t\right),
$$

whose solution is $\widetilde{f}(t)=\frac{e^{\alpha t}}{\sqrt{\cos \omega t}}$, from which we obtain the function $f(t)$ as

$$
f(t)=\sqrt{\frac{m \omega}{2 \pi i \hbar} \cot \omega t} \frac{e^{\alpha t}}{\sqrt{\cos \omega t}}=e^{\alpha t} \sqrt{\frac{m \omega}{2 \pi i \hbar \sin \omega t}} .
$$

As in the case of the free particle by taking the step III we can find the numerical value of the parameter $\alpha$. However, in the limit $\omega \rightarrow 0$, the above expression takes the form of the function $f(t)$ for the free particle for which we have already obtained $\alpha=0$. This completes our calculations to evaluate the $f(T)$ function for harmonic oscillator as

$$
f(T)=\sqrt{\frac{m \omega}{2 \pi i \hbar \sin \omega T}}
$$

\section{Summary}

In this letter we have investigated the issue of path integral for a class of systems whose Lagrangian has a general quadratic form. We showed that the transition amplitude for such systems is proportional to $e^{\frac{i}{\hbar} S\left[q_{c l}\right]}$ and the coefficient function $f(T)$ depends only on the initial and final times as $T=t_{f}-t_{i}$ for which we have obtained a formula in terms of a path integral of the variation function around the classical trajectory. In the rest of the paper we proposed a systematic algebraic method to evaluate the function $f(T)$. The presented method is based on three steps through which we showed that this function should satisfy a first order differential equation whose initial condition is coded in itself. We applied the mentioned method to the cases of free particle and harmonic oscillator and showed that the resulting propagators are exactly the same as are obtained in usual ways in path integral formulation of quantum mechanics.

\section{Acknowledgement}

The author is grateful to the research council of IAU, Tonekabon Branch, for financial support.

\section{References}

1. R.P. Feynman and A.R. Hibbs, Quantum Mechanics and Path Integrals, McGraw-Hill, New York, 1965

2. P.A.M. Dirac, The Principles of Quantum Mechanics, Oxford University Press, 1958

P.A.M. Dirac, Lectures on Quantum Mechanics, Dover Publications, New York, 1964

3. A. Das, Field theory, a path integral approach, Word Scientific Publishing, Singapore, 1993

4. R. Shankar, Principles of Quantum Mechanics, Plenum Press, New York, 1994 